\title{
Application of Multilayer Neural Network in Sports Psychology
}

\author{
Hongling Yang $\mathbb{D}$ \\ Guangdong Industry Polytechnic, Guangzhou 510300, China \\ Correspondence should be addressed to Hongling Yang; 2010104085@gdip.edu.cn
}

Received 11 October 2021; Revised 28 November 2021; Accepted 13 December 2021; Published 7 January 2022

Academic Editor: Rahman Ali

Copyright (c) 2022 Hongling Yang. This is an open access article distributed under the Creative Commons Attribution License, which permits unrestricted use, distribution, and reproduction in any medium, provided the original work is properly cited.

\begin{abstract}
The research on multilayer neural network theory has developed rapidly in recent years. It has parallel processing capabilities and fault tolerance and has aroused the interest of many researchers. The neural network has made great progress in the field of control, especially in model identification and control. It has been quickly applied in the fields of device design, optimized operation, and fault analysis and diagnosis. Neural network control, as an automated control technology in the 21st century, has been fully proved by theories and practices at home and abroad, and it is very useful in complex process control. Sports psychology is a discipline that studies the psychological characteristics and laws of people engaged in sports, and it is also a new development in sports science. The main task of sports psychology is to study people's psychological processes when participating in sports, such as feeling, perception, appearance, thinking, memory, emotion, and characteristics of will and its role and significance in sports. An important feature of multilayer neural networks is to achieve results that match the expected output through network learning. It has strong self-learning, self-adaptability, and fault tolerance. The multilayer neural network system evaluation method is unique with its extraordinary ability to deal with complex nonlinear problems and does not involve human intervention. This article presents a multilayer neural network algorithm, which classifies the samples of athletes, and studies the physical education training process, the psychological characteristics of related personnel in sports competitions, such as the psychological characteristics of the formation of sports skills, and the psychological training of athletes before the game.
\end{abstract}

\section{Introduction}

Multilayer neural network theory is an international frontier research field that has developed rapidly in recent years. It has parallel processing capabilities and fault tolerance and can approximate arbitrary nonlinear functions, self-learning, self-adaptation, and associative memory functions $[1,2]$. It is very attractive that the multilayer neural network has aroused the interest of many researchers and has made significant progress in various fields. In the application of neural network, there is no need to consider the internal mechanism of the process or phenomenon. Some highly nonlinear and highly complex problems can be handled well. Therefore, the neural network has made great progress in the field of control, especially in model identification and control. It has been quickly applied in the fields of device design, optimized operation, and fault analysis and diagnosis. Neural network control, as an automated control technology in the 21st century, has been fully proved by theories and practices at home and abroad, and it is very useful in complex process control. Sports psychology is a discipline that studies the psychological characteristics and laws of people engaged in sports, and it is also a new development in sports science [3-5]. It is closely related to sports science, sports sociology, exercise physiology, sports training theories and methods, and other sports theories and methods. The main task of sports psychology is to study people's psychological processes when participating in sports, such as feeling, perception, appearance, thinking, memory, emotion, and characteristics of will and its role and significance in sports. It also studies the characteristics of personality, initiative, and temperament when people participate in various sports and the influence of sports on personality characteristics. Multilayer neural network is a cutting-edge research field used to simulate the structure and intelligent characteristics of the human brain. An important feature of it is to achieve results that match the expected output through network learning. It has strong self-learning, 
self-adaptability, and fault tolerance in addition to the ability to store memory. The multilayer neural network system evaluation method is unique with its extraordinary ability to deal with complex nonlinear problems. This evaluation method is faithful to objective reality and does not involve any human intervention [6]. Sports psychology is the science of applying the principles of psychology to sports or exercise multilayer neural network scenarios. It studies the influence of psychological factors and emotional factors on sports performance multilayer neural networks and exercise effects. It also studies participation in sports and exercise. For a long time, we have used multilayer neural networks to analyze psychological and emotional factors, and the research on multilayer neural network in sports psychology in our country has mainly focused on three aspects: competitive sports psychology, sports education multilayer neural network theory, and mass exercise psychology [4, 7-9]. The directions are discussed separately. With the increasing expansion of the research object of multilayer neural networks and the continuous updating of research methods, the cross-penetration between the three research directions of multilayer neural networks has become more and more frequent. Multilayer neural network is a kind of image that shows the relationship between the development process and structure of scientific knowledge of multilayer neural network based on the knowledge domain as the research object [10].

The scientific research communities of sports colleges and normal universities have become the main driving force for the development of sports psychology [11-13]. About multilayer neural networks, the research hotspots of sports psychology in China mainly focus on improving the mental health of various groups. The research objects and research hotspot time zone continue to expand. It presents the two stages of exploration and anti-multilayer neural network thinking and expansion and innovation. The research content includes psychological training, skill learning, social adaptation, physical education process, physical activity, and exercise rehabilitation.

In the application of neural network, there is no need to consider the internal mechanism of the process or phenomenon. Some highly nonlinear and highly complex problems can be handled well. Therefore, the neural network has made great progress in the field of control, especially in model identification and control. It has been quickly applied in the fields of device design, optimized operation, and fault analysis and diagnosis. Multilayer neural network is based on the basic understanding of human brain neural network, using mathematical methods and from the perspective of information processing to abstract the human brain neural network and establish a certain simplified mathematical model [14]. It is a large-scale parallel distributed processor composed of simple processing units, which naturally has the characteristics of storing experience knowledge and making it available. Neural network control, as an automated control technology in the 21st century, has been fully proved by theories and practices at home and abroad, and it is very useful in industrial complex process control. However, the industrial field needs advanced control methods and urgently needs engineering and practical neural network control methods. Therefore, studying the application of neural networks in control is of great significance to the improvement of the automation level of our country and the economic benefits of enterprises.

The rapid development of science and technology and the tremendous progress of society have put forward new and higher requirements for sports psychology. Multilayer neural networks have encountered some problems in the application of sports psychology. First of all, the analysis and design of these systems in sports psychology are based on accurate system mathematical models. However, actual systems are generally difficult due to factors such as nonlinearity, uncertainty, time varying, and incompleteness. Obtain accurate mathematical models; secondly, when studying these systems, some assumptions are generally first put forward, and these assumptions are often not completely consistent with the reality in the application, which inevitably leads to larger errors. Therefore, for many years, sports psychology researchers have been looking for solutions, and the application of multilayer neural networks has brought a dawn. Multilayer neural network is one of the three major areas of intelligent control. It has been paid attention to by many scholars since its birth and has made great progress in prediction, safety monitoring, control, and pattern recognition. In our country, through the long-term efforts of scientific researchers, some aspects of multilayer neural network theory have approached or reached the world's advanced level, but there is a huge disconnect between these advanced theories and engineering practice, and the research has engineering practical intelligence [15].

\section{Related Work}

The keywords in the retrieved documents generally reflect the main content of the document. If the same keywords appear frequently in the literature of a certain research field, we generally tend to think that these keywords or topic words can reflect hot topics in this research field. The network node (Node type) selects the keyword (Keyword) and obtains the core keyword cooccurrence network map in the field of sports psychology research in my country. Among them, each circular node represents a keyword in sports psychology research. The size of the circle is proportional to the frequency of the keyword. The depth of the circle represents different years, and the level change shows the evolution of the keyword from far to near with the years. The line between nodes indicates that there is an association between two keywords, and the width of the line indicates the degree of affinity between the keywords. Keywords with relatively high frequency and centrality jointly show the hot topics in the field of sports psychology research in my country from 2008 to 2017 (Table 1). The keyword cooccurrence map reflects important nodes in the field of sports psychology in my country $[16,17]$. It can be seen that the frequency of occurrence of larger nodes is 279 times for mental health, 202 times for college students, 186 times for physical education, 101 times for physical exercise, 89 times for school physical education, 81 times for influencing 
TABLE 1: Keywords overview.

\begin{tabular}{|c|c|c|c|c|}
\hline & \multicolumn{2}{|c|}{ High-frequency keywords } & \multicolumn{2}{|c|}{$\begin{array}{c}\text { High school psychology } \\
\text { keywords }\end{array}$} \\
\hline & Keyword name & Frequency & Keyword name & Centrality \\
\hline 1 & $\begin{array}{l}\text { Psychological } \\
\text { health }\end{array}$ & 279 & Undergraduate & 0.26 \\
\hline 2 & Undergraduate & 202 & $\begin{array}{l}\text { Psychological } \\
\text { health }\end{array}$ & 0.21 \\
\hline 3 & $\begin{array}{l}\text { Physical } \\
\text { education }\end{array}$ & 186 & Physical exercise & 0.19 \\
\hline 4 & Physical exercise & 101 & $\begin{array}{c}\text { Athletic } \\
\text { psychology }\end{array}$ & 0.18 \\
\hline 5 & School physical & 89 & Influence factor & 0.17 \\
\hline 6 & $\begin{array}{l}\text { Influencing } \\
\text { factor }\end{array}$ & 81 & Sports education & 0.16 \\
\hline 7 & Athletes & 70 & $\begin{array}{l}\text { Physical } \\
\text { education }\end{array}$ & 0.16 \\
\hline 8 & $\begin{array}{l}\text { Physical } \\
\text { education }\end{array}$ & 75 & Physical training & 0.15 \\
\hline 9 & Sports activities & 70 & $\begin{array}{l}\text { Rehabilitation } \\
\text { training }\end{array}$ & 0.12 \\
\hline 10 & Living quality & 53 & Rehabilitation & 0.12 \\
\hline
\end{tabular}

factors, 77 times for athletes, and 75 times for physical education. We have 10 times, 70 times physical activity, and 53 times the quality of life. Based on the above statistical results, the research objects in the field of sports psychology in my country have been expanding in the past 10 years, including college students, athletes, the elderly, teachers, and youth groups; research hotspots are mainly concentrated in the mental health of college students or elderly groups and physical education $[18,19]$.

In the past, the discourses on sports psychology were mostly discussed from the three directions of competition, sports, and mass exercise. It is generally believed that the research on competitive sports psychology belongs to the mainstream research category. The evolution of the size and level of the nodes in Figure 4 clearly shows that the three directions are the development of public exercise psychology and that physical education psychology has been greatly developed. The development of public exercise psychology has been very rapid in the past 10 years. Since 2009, five academic conferences on physical exercise and mental health have been held. In 2019, the 6th Academic Conference on Physical Exercise and Mental Health will be held in Sichuan, highlighting the results of the implementation of the "Mass Fitness Program," which is also in line with the current development trend of "Healthy China" [20-22].

Xuet al. [23] used multilayer perceptron and CNN to build a sportswear retailing forecast model. They collect data from some offline sports stores to train the model and propose a novel loss function to improve the forecast accuracy rate. They define influencing factors including time series sale data, features of the products, strategy used for distribution, and size of the shop. They claimed a higher accuracy of $65 \%$ compared to other systems with only about $20 \%$ accuracy rates. Aldosary and Alrashdan [24] used artificial neural networks-based model to predict whether gym members are going to end their agreement with the gym and join another one. They applied multilayer perceptron using backpropagation with an emphasis on feature selection. They concluded from their study that implementing psychological concept of habit formation serves as a link for introducing effective artificial neural network-based model into the fitness strategies. Nadeem et al. [25] conclude that physical activity recognition is an essential tool for smart health monitoring and for fitness exercises. They propose a unified framework to explore multidimensional features with the help of a fusion of body part model and discriminant analysis, using the features for human pose estimation. The features are processed with Markov model as a recognition model. They use various datasets and achieve an accuracy of up to $90 \%$.

The research of young people and ethnic minorities has also received increasing attention. It can be seen from this that in the course of development and evolution of sports psychology research in my country, research topics have been continuously enriched, research has continued to have meticulous and in-depth content, research methods have continued to innovate, the relationship with the parent subject has become increasingly close, interdisciplinary research has increased, and more attention has been paid to cognitive perspective to examine the psychological impact.

\section{Method}

3.1. Multilayer Neural Network Data Source. The data required is retrieved and collected using the China National Knowledge Infrastructure (CNKI) database platform. In the visual analysis of the scientific knowledge map, the data directly determines the results of the analysis. Therefore, the first important problem to be solved in this research is how to make the retrieved data as comprehensive and accurate as possible to include the research results in the field of sports psychology in China. After the combination and debugging of multiple retrieval methods, such as the classification number of the Chinese Library Classification, and a comparative analysis of the retrieval results, it was finally determined that the retrieval method was set as the subject word "sport" or "sports" according to the traditional classification method of sports psychology research or "sports" or "exercise" with "psychology," the search condition is set to "precise," the time span is set to 2008 to 2017, the document source category is set to "core journals," and the retrieval date is January 23,2018 . On the $23 \mathrm{rd}$, a total of 3675 related index documents were retrieved. Then, some documents were manually deleted such as "meeting notices," "call for papers," and documents that are obviously not in the field of sports psychology to improve the effectiveness of the search data. Finally, 3260 retrieved documents were confirmed as the source of the research data for analysis and processing.

3.2. Ideas and Learning Rules of Multilayer Neural Network Algorithms. The basic idea of the multilayer neural network algorithm is that the learning process is composed of two processes, the forward propagation of the signal and the 
backward propagation of the error. In the forward propagation, the input samples are passed from the input layer, processed by each hidden layer time slicing by layer, and then passed to the output layer. If the actual output of the output layer does not match the expected output, it will enter the error backward propagation stage. It is mainly through the gradient descent method that the weight is modified, so that the total error function is minimized. Suppose the network has a total of $L$ layers and $N_{1}$ is the number of neurons in the first layer, where $l=1,2, \ldots, L$. The total number of samples entered is $P$.

The sample $P$ input vector is as below:

$$
X_{p}=\left(x_{p 1}, x_{p 2}, \ldots, x_{p n}\right)^{r} .
$$

The expected output vector is as below:

$$
d_{p}=\left(d_{p 1}, d_{p 2}, \ldots, d_{p k}, \ldots, d_{p n}\right)^{r} .
$$

The actual output vector is as below:

$$
O_{p}^{(1)}=\left(O_{p 1}^{(1)}, O_{p 2}^{(1)}, \ldots O_{p k}^{(1)}, \ldots, O_{p n}^{(1)}\right)^{r} .
$$

3.3. Research Methods of Multilayer Neural Networks. CiteSpace III visualization tool based on JAVA platform is used to draw scientific knowledge graph. The CiteSpace III visual analysis software was developed by Chen Chaomei, a professor at the School of Information Science and Technology of Drexel University and a Yangtze River Scholar Chair Professor at Dalian University of Technology. It can draw a huge amount of raw data and visually present it in graphics or images. The research hotspots, development trends, and sudden changes in the dynamic development network structure of a certain subject knowledge field provide support for clearly understanding and grasping the development trend of the subject. The preprocessed 3260 literature documents are imported into CiteSpace III visual analysis software, the time span is set to 2008-2017, the time zone division (time slicing) is set to one stage per year, and the critical path algorithm (Pathfinder) is used for institutions. The author, keyword, and other functions are analyzed and processed; we conducted cluster analysis on the cooperation relationship between core academic research institutions and core academic researchers; the annual number of articles and other data are used for mathematical statistics.

3.4. Program Realization of Multilayer Neural Network Algorithm. Experimental method is an important method in psychology research. Compared with other research methods, experimental method is more able to test causality and explore the laws between things. In experimental method, the researchers use instruments and equipment, artificially control and intervene in the research object according to the purpose of the subject, eliminate interference factors, highlight the key points, and observe the experimental object under favorable conditions. The measurement method is a method of collecting data that uses tools to measure the characteristics of the human body in sports science research. Because of its quantification, error, and unit consistency uncertainty, it determines its status in sports research. The measurement method is divided into self-made scale and ready-made scale. Self-made scales are some items compiled by researchers according to the problems to be studied. In the use of self-made scales by Chinese researchers, most of them lack the proof of the validity of the scale, and the basic principles of psychological measurement are not used for demonstration. The readymade scale is tested by time and practice and has a certain degree of representativeness and practicability. Tan Xianming and Chen Gengchang's "Analysis of the Application Status of Psychological Scales in the Field of Sports Psychology" in the past ten years included sports science, sports journals, China Sports Science and Technology, and psychological sciences. Journal of Beijing Sport University, Journal of Wuhan Institute of Physical Education, and Shanghai Institute of Physical Education 670 papers on sports psychology in 14 journals including the Chinese Journal of Analytical Research have been conducted. It is found that only $25 \%$ of the papers using psychological scales are used by most researchers. The scales used by most researchers are borrowed from other fields of psychology. Very few of them have compiled their own scales, and the most used scales are sixteen cartels. We collected personality factor test (16PF), trait anxiety questionnaire, Sports Competition Anxiety Scale (SCAT), etc. The programming steps of the multilayer neural network algorithm are as follows: initialize the weight matrix $W(1), W(2), \ldots, W(L)$ to assign random numbers, set the sample pattern counter $\mathrm{P}$ and the training times counter $q$ to 1 , set the error $E$ to 0 , set the learning rate to a number within $0-1$, and set the accuracy $E$ achieved after the network training to a small positive number; input the training sample pair, and calculate the output of each layer; calculate the network output error; calculate according to the formula the error signal of each layer; adjust the weight of each layer according to the formula; check whether a rotation training is completed for all samples. If $p<P$, the counters $p$ and $q$ increase by 1 , return the input training sample pair, and calculate the output of each layer; otherwise, check whether the total error of the network meets the accuracy requirements. If $E<E_{\text {min }}$, the training ends; otherwise, $E$ is set to $0, p$ is set to 1 , and return to the step of inputting the training sample pair and calculating the output of each layer.

\section{Experiment and Result Analysis}

The research of sports psychology has been a hot area of psychology at home and abroad in the past few decades. In China, the "Sports Psychological Characteristics Research" project that began in 1980 has driven the continuous exploration of most sports psychologists in China. A variety of domestic and foreign psychological characteristics, research tools, and methods have been introduced and applied in volleyball gymnastics and table tennis. We choose sports psychology in different sports as our choice and evaluation, such as track and field, shooting, swimming, football, weightlifting, and badminton boating. In foreign countries, 
since the 1960s and 1970s, the research works on sports psychological characteristics are also conducted one after another. Researchers have tried to find some universally significant and stable "success factors" through comparative studies of excellent athletes and the general population, to provide scientific basis for athletes' selection, diagnosis, training, competition, and other practical activities. In this way, the enthusiasm for research has enriched the research on the psychological characteristics of sports, both in experience and in theory, and the connotation of this research field has also been continuously expanded.

On the one hand, in the field of sports psychology, some new theories and models that are completely different from the traditional have emerged in recent years, which have also challenged the research models and research ideas based on traditional theories; on the other hand, in sports psychology within the scope of learning, the existing research conclusions related to athletes' personality and psychological characteristics have not been effectively verified in sports selection and sports competition practice, and the actual benefits of most studies still need to be tested.

This experiment will compare the cognitive processing ability (digital calculation) and physiological (heart rate) trends of gymnasts of different levels (master group, general athlete group, and nongymnast group) in the stress and passion environment. The changes of the two indicators in the three groups of subjects in a calm state and in a stressful (interference) situation are shown in Tables 2 and 3.

In the table, $X$ represents the average value of the difference between the calculation ability of the three groups of test subjects in a stressful environment (under laboratory conditions) and the calculation ability in a calm state. The smaller the difference is, the less the calculation ability of this group of subjects is affected by the stress factors. The results in the table show that excellent gymnasts (master level) are significantly better than the other two groups in the degree of influence of stress factors.

The $X$ in the table represents the average value of the difference between the heart rate of the test subject in a stressful state (under laboratory conditions) and the heart rate in a calm state. A smaller difference indicates that the changes in the heart rate of this group of subjects are less affected by stress factors. Table 3 shows that the change of heart rate in the elite athlete group is smaller than that of the other two groups at the level of 0.1 or close to 0.1 .

To explain the reasons for the variation of the above two results, the study separately examined the influence of factors such as age, education level, exercise level, and training years on the calculation ability and the trend of heart rate changes. The results show that the above factors have no significant effect on the changing trends of the two indicators in the three groups of subjects in different situations. Therefore, this observed cross-situational stability can be attributed to individual personality characteristics. In this study, it was named "anti-interference ability." However, according to the CAPS theory, this research result provides a preliminary example for the application of cognitive-emotional system theory in the field of sports psychology.
TABLE 2: The tendency to change in a calm state.

\begin{tabular}{lccc}
\hline Group & Juvenile mobilization & Letterman & Nonathlete \\
\hline$n$ & 72 & 100 & 231 \\
$X$ & 0.27 & -0.68 & 0.28 \\
$S$ & 2.7 & 2.78 & 2.64 \\
$t$ & & 2.21 & 3 \\
\hline
\end{tabular}

TABLE 3: The tendency to change in stressful situations.

\begin{tabular}{lccc}
\hline Group & Juvenile mobilization & Letterman & Nonathlete \\
\hline$N$ & 68 & 92 & 225 \\
$X$ & 2.26 & 1.32 & 2.99 \\
$S$ & 5.75 & 5.29 & 7.96 \\
$T$ & & 1.54 & 1.86 \\
\hline
\end{tabular}

This experiment uses the four indicators of accuracy, precision, recall, and $F 1$ score to measure the predictive ability of the classification model. These indicators are common detection and classification indicators. These common metrics are all related to the confusion matrix (also called the error matrix). The confusion matrix evaluates the model's classification of the psychological change dataset.

$\mathrm{TP}$ represents the number of samples whose true results and predicted results are true; FN represents the number of samples whose true results are true and predicted results are false; FP represents the number of samples whose true results are false and predicted results are true; TN represents the number of samples whose both true results and predicted results are true. According to Table 3, the calculation formula of accuracy rate is shown in (4), the calculation formula of accuracy is shown in (5), and the calculation formula of recall rate is shown in (6).

$$
\begin{aligned}
\text { Accuracy } & =\frac{\mathrm{TP}+\mathrm{TN}}{\mathrm{TP}+\mathrm{TN}+\mathrm{FP}+\mathrm{FN}}, \\
\text { Precison } & =\frac{\mathrm{TP}}{\mathrm{TP}+\mathrm{FP}}, \\
\text { Recall } & =\frac{\mathrm{TP}}{\mathrm{TP}+\mathrm{FN}} .
\end{aligned}
$$

If there are only precision and recall values, it is impossible to compare the quality of the classification model, so the index $F 1$ score is required. The $F 1$ score is a judgment index that combines the precision value and the recall value. Through the calculation of the $F 1$ score, a value between 0 and 1 will be obtained. The closer to 1 , the better the classification model. At this time, you can compare the classification models. The definition formula of $F 1$ is as follows:

$$
F 1=2 * \frac{\text { Precision } * \text { Recall }}{\text { Precision }+ \text { Recall }} \text {. }
$$

In the arrhythmia classification model, after disrupting the order of all ECG samples, 16033 ECG datasets are selected as the training set of the classification model, and other ECG datasets are used as the test set of the classification model. In the training process, the accuracy rate of the classification model and the graph of the increase of the loss function value with the 


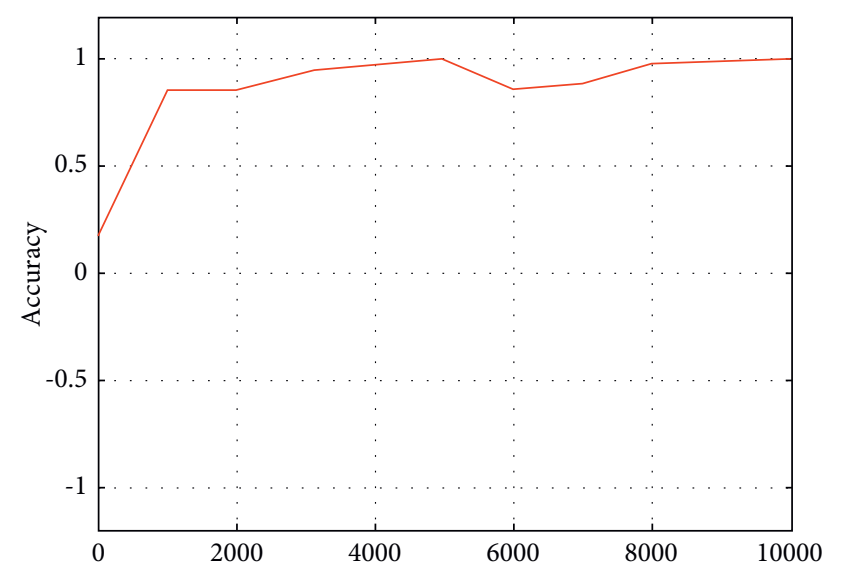

Figure 1: Changes in accuracy during training.

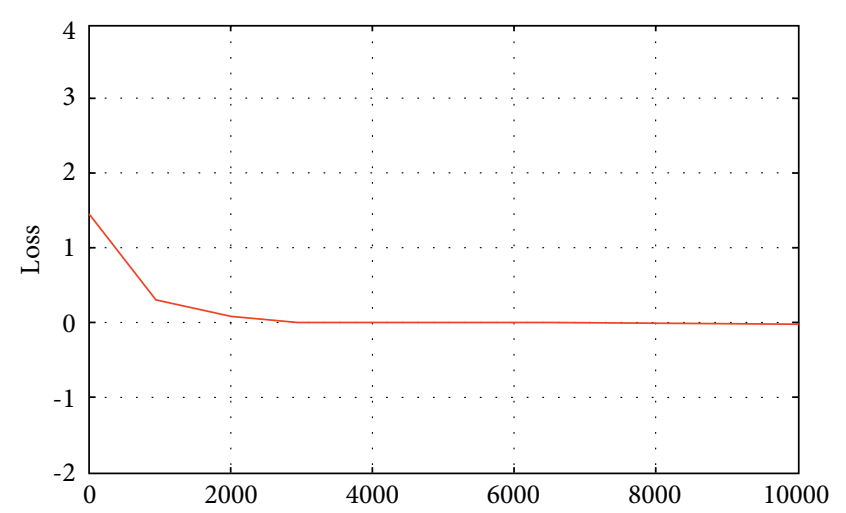

Figure 2: Change in loss value during training.

number of iterations are recorded, as shown in Figures 1 and 2. It can be seen from Figure 35 that the loss function value reaches convergence at about 3000 steps. This has two main reasons. First, the ECG signal belongs to one-dimensional data, which has a relatively simple data form, and the signal data points are less than 300. The second is the CNN-BiLSTM model. Although the classification model has many layers, the classification model reduces the number of parameters and improves the accuracy (shown in Figures 1 and 2).

After the classification model is trained, the classification results obtained on the test set are shown in Figure 3, and the confusion matrix obtained on the training set is shown in Table 4. It can be seen from Figure 3 that the CNN-BiLSTM classification model can classify different arrhythmias including normal heartbeat beats. Although normal beats and abnormal beats are sometimes confused, most of the beats can be accurately detected.

\section{Conclusion}

This paper uses multilayer neural networks to build models and build neural network control systems. We select multiple network types, write neural network software, and write interfaces that are integrated and run with multiple configuration software to improve neural network software. Different types of artificial neural networks are used to identify

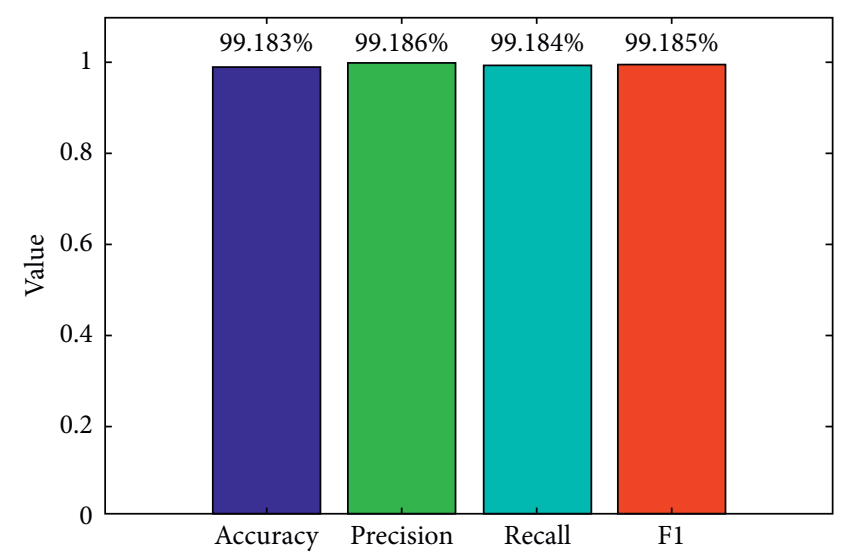

Figure 3: Classification result.

TABle 4: Confusion matrix.

\begin{tabular}{lcccc}
\hline Group & $N$ & $L$ & $R$ & $V$ \\
\hline$N$ & 3996 & 2 & 0 & 0 \\
$L$ & 13 & 3972 & 11 & 21 \\
$R$ & 9 & 21 & 3967 & 0 \\
$V$ & 3 & 51 & 0 & 3968 \\
\hline
\end{tabular}

complex objects and analyze the identification results. Neural network is used to adjust PID parameters to control complex objects and improve the algorithm. The PID neural network controller is directly used to construct an artificial neural network control system to directly control complex objects without a model. This experimental model uses multilayer network neural algorithms to carry out applied research on sports psychology, which is useful for further research on the characteristics and laws of human psychological processes in sports and the relationship between human personality differences and sports. The short-term and long-term effects of the psychological process and personality characteristics, the study and mastering of sports knowledge, the formation of sports skills, the psychological laws of skill training, and the study of people's mental state in sports competitions have very important practical reference significance. Constantly increasing and improving system performance will play an increasingly important role in competitive sports.

\section{Data Availability}

The datasets used and analyzed during the current study are available from the author upon reasonable request.

\section{Conflicts of Interest}

The author declares no conflicts of interest.

\section{References}

[1] M. Ahr, M. Biehl, and E. Schlösser, Multilayer Neural Networks, Springer International Publishing, New York USA, 2004.

[2] K.. Vera, "Kolmogorov's theorem and multilayer neural networks," Neural Networks, vol. 5, no. 3, pp. 501-506, 1992. 
[3] P. Agrawal, R. Girshick, and J. Malik, "Analyzing the performance of multilayer neural networks for object recognition," in Proceedings of the Computer Vision - ECCV 2014, September 2014.

[4] J. Annett, "Sports psychology," Journal of Sports Sciences, vol. 9, no. 1, pp. 1-2, 1991.

[5] C. S. Cheng, "A multi-layer neural network model for detecting changes in the process mean," Computers of Industrial Engineering, Pergamon Press, vol. 28, no. 1, pp. 51-61, 1995.

[6] M. Kaminski and O. T. Kowalska, "FPGA implementation of the multilayer neural network for the speed estimation of the two-mass drive system," IEEE Transactions on Industrial Informatics, vol. 7, no. 3, 2011.

[7] M. Bryner, "My two mommies," Journal of Sports Psychology, vol. 28 , no. 18 , p. 102, 2005.

[8] L. Rosellini, "Delayed allergic reaction to penicillin in oil and wax treated with procaine intravenously," Sports psychology, vol. 102 , no. 22, p. 58, 1987.

[9] W. D. Ren, "Development of sports psychology related to competitive sports," Journal of Wuhan Institute of Physical Education, 2005.

[10] R. N. Singer, "Sports psychology," American Corrective Therapy Journal, vol. 29, no. 4, p. 115, 1975.

[11] C. M. Pierce, "On the importance of sports psychology," Journal of Clinical Psychology, vol. 36, no. 1, pp. 366-367, 1980.

[12] C. Heaney, "The impact of sport psychology education on the practice of physiotherapists," British Journal of Sports Medicine, 2013.

[13] J. Guasch, "The lymph node type as a rare type of extramedullary plasmocytoma," Sangre, vol. 11, no. 2, pp. 249-251, 1966.

[14] K. Lang, Experimental Research on Node-type Heat-Exchange Ele Ments, JOURNAL OF NORTHEASTERN UNIVERSITY, 1995.

[15] Q. Han, Q. Zou, and H. N. University, Analysis on Research Hotspot and Evolution about Leisure Sports, Journal of Henan Normal University (Natural Science Edition), Changsha, Hunan Province, 2015.

[16] J. Pei, K. Zhong, J. Li, I. Xu, and X. Wang, "ECNN: evaluating a cluster-neural network model for city innovation capability," Neural Computing \& Applications, vol. 206, pp. 1-13, 2021.

[17] L. Chao, F. Li, and S. Xiang, "Research hotspot and progression of national economic accounting in China--Based on knowledge map and visualized analysis," China Economic Statistics Quarterly, 2013.

[18] I. Ciuca and E. Jitaru, "On the three-layer neural networks using sigmoidal functions," in Proceedings of the International Work-conference on Artificial Neural NetworksSpringer, Berlin Heidelberg, 1999.

[19] K. Zhong, Y. Wang, J. Pei, S. Tang, and Z. Han, "Super efficiency SBM-DEA and neural network for performance evaluation," Information Processing \& Management, vol. 58, no. 6, Article ID 102728, 2021.

[20] A. Kowalczyk, "Can multilayer mapping networks with finite number of real parameters harness the computational power of Kolmogorov's representation theorem," in Proceedings of the IEEE International Joint Conference on Neural Networks, November 1991.

[21] Sc Mirek Karn Csc, D. K. Warwickc, and K. Vera, Recent Results and Mathematical Methods for Functional Approximation by Neural Networks, Springer, London, 1998.
[22] M. A. Sartori and P. J. Antsaklis, "A simple method to derive bounds on the size and to train multilayer neural networks," IEEE Transactions on Neural Networks, vol. 2, no. 4, pp. 467-471, 1991

[23] J. Xu, Y. Zhou, L. Zhang, J. Wang, and D. Lefloch, "Sportswear retailing forecast model based on the combination of multilayer perceptron and convolutional neural network," Textile Research Journal, Article ID 00405175211020518, 2021.

[24] M. Aldosary and A. Alrashdan, "Churn prediction for gym members using artificial neural networks assisted with the psychological concept of habit formation in the fitness industry,".

[25] A. Nadeem, A. Jalal, and K. Kim, "Accurate physical activity recognition using multidimensional features and Markov model for smart health fitness," Symmetry, vol. 12, no. 11, p. $1766,2020$. 